\title{
The psychological impact of injury: effects of prior sport and exercise involvement
}

\author{
Lynne H Johnston, Douglas Carroll
}

\begin{abstract}
Objectives-To test the assumption that the psychological impact of injury varies with involvement in sport and exercise, and that those who are more involved in sport and exercise before injury would experience greater negative affect and retarded recovery.

Method-Patients attending for physiotherapy completed a battery of questionnaires including measures of mood and perceived recovery, at the beginning, middle, and end of formal rehabilitation. Complete data were available for 93 patients. Results-Those who were more involved in sport and exercise before injury registered higher levels of confusion and perceived their recovery to be less, possibly reflecting greater information needs and a greater mismatch between current status and that before injury in the athletic sample. Reported negative affect did not vary with sport and exercise involvement. Conclusions-Incapacitation for those not involved in sport and exercise before injury may have much the same affective impact as it does for those with considerable involvement. However, those with considerable involvement did report higher levels of confusion and perceived their recovery to be less towards the end of rehabilitation. This suggests that it may be important to assess affective reactions and perceived recovery during the re-entry phase.

(BrF Sports Med 2000;34:436-439)
\end{abstract}

Leisure and Sport

Research Unit, Cheltenham and Gloucester College of Higher Education, Cheltenham

GL50 4AZ, United

Kingdom

L H Johnston

School of Sport and Exercise Sciences,

University of

Birmingham,

Birmingham B15 2TT,

United Kingdom

D Carroll

Correspondence to: L H Johnston, Leisure and Sport Research Unit, Cheltenham and Gloucester College of Higher Education, Swindon Road, Cheltenham GL50 4AZ, United Kingdom email:

Ljohnston@chelt.ac.uk

Accepted for publication 12 July 2000
Keywords: injury; psychological impact; physiotherapy; rehabilitation; recovery

It is estimated that 29.7 million sports injuries occur annually in England and Wales at a total cost in terms of treatment and lost working days of $£ 991$ million. ${ }^{12}$ Injury to those involved in sport and exercise has also been reported to have a substantial psychological impact. Quantitative studies examining differences between injured and uninjured athletes have disclosed greater negative affect, lower self esteem, and higher levels of depression and anxiety among the injured athletes. ${ }^{3-6}$ Comparisons of athletes before and after injury have indicated greater mood disturbance, lowered self esteem, and increased depression after injury. ${ }^{47}$ Studies that have charted emotional state over the period of injury have generally found a move from negative to positive affect over time..$^{7-11}$ The degree of this shift appears to be dependent on actual and perceived rehabilitation success. ${ }^{9}$ Recent qualitative studies also attest to the negative impact of injury in athletes, as well as the shift in affect over time. ${ }^{12-14}$

A general assumption within much of the sport injury literature, which is increasingly reflected in treatment provision, is that athletes and non-athletes differ radically in the way they respond to injury. ${ }^{15} 16$ For example, concepts borrowed from analysis of the bereavement process have been applied to account for the emotional impact of athletic injury. ${ }^{9}$ In addition, those who have championed a cognitive appraisal account of psychological responses to injury argue that the greater the psychological investment in sport and exercise, or the more committed the person is ${ }^{17}$, then the greater the stress of injury. ${ }^{15}$ Further, the well recognised psychological benefits of exercise may be jeopardised by injury, with consequences for negative affects such as anxiety and depression. ${ }^{18} 19$

Nevertheless, the assumption that the psychological impact of injury is related to psychological and or physiological investment in sport and exercise has received little in the way of formal study. To date, there are only four published reports of sports involvement as a factor in injury impact. ${ }^{11}{ }^{20-22}$ Although the results of these studies are indicative, they can hardly be regarded as offering definitive evidence that the psychological response to injury is related to involvement in sport and exercise. With one exception, ${ }^{11}$ these previous studies have used indirect measures of sports and exercise activity. Further, responses have been assessed to hypothetical rather than real injuries ${ }^{20}$ and, in other instances, at some considerable time after the injury was sustained. ${ }^{21}$ In addition, none of these studies included repeat assessments at standard temporal locations during rehabilitation.

This study was undertaken to provide a more rigorous test of the hypothesis that the psychological impact of injury will vary with involvement in sport and exercise, and that those who are more involved will experience greater negative affect consequent to injury. As a consequence, but also as a result of the need to regain their previous high level of physical functioning, it was also expected that those with a greater involvement in sport before injury would also perceive their recovery to be less. Finally, given that the affective impact of injury changes over the course of rehabilitation, it was considered important to perform repeated assessments.

\section{Methods}

PARTICIPANTS

Physiotherapists in 40 NHS hospitals, community units, or health centres, 36 private hospitals and physiotherapy clinics, and 23 
Table 1 Mean (SD) scores for all outcome variables across time

\begin{tabular}{|c|c|c|c|c|c|}
\hline & Time 1 & Time 2 & Time 3 & $F$ & Post hoc \\
\hline Anger & $30.59(30.79)$ & $17.38(19.57)$ & $8.85(13.95)$ & $F(2,180)=30.71, \mathrm{p}<0.01$ & $\mathrm{~T} 1>\mathrm{T} 2>\mathrm{T} 3$ \\
\hline Anxiety & $53.55(25.57)$ & $27.86(21.47)$ & $17.42(20.66)$ & $F(2,180)=72.98, \mathrm{p}<0.01$ & $\mathrm{~T} 1>\mathrm{T} 2>\mathrm{T} 3$ \\
\hline Confusion & $22.37(25.98)$ & $15.98(19.80)$ & $8.73(14.92)$ & $F(2,180)=14.28, \mathrm{p}<0.01$ & $\mathrm{~T} 1>\mathrm{T} 2>\mathrm{T} 3$ \\
\hline Depression & $40.30(26.78)$ & $21.80(22.94)$ & $12.54(19.71)$ & $F(2,180)=48.56, \mathrm{p}<0.01$ & $\mathrm{~T} 1>\mathrm{T} 2>\mathrm{T} 3$ \\
\hline Energy & $43.17(24.14)$ & $56.18(20.55)$ & $67.57(22.99)$ & $F(2,180)=38.64, \mathrm{p}<0.01$ & $\mathrm{~T} 1<\mathrm{T} 2<\mathrm{T} 3$ \\
\hline Fatigue & $41.95(24.05)$ & $32.03(22.36)$ & $25.28(23.54)$ & $F(2,180)=17.19, \mathrm{p}<0.01$ & $\mathrm{~T} 1>\mathrm{T} 2>\mathrm{T} 3$ \\
\hline Recovery & $42.80(22.77)$ & $63.56(18.18)$ & $78.63(17.18)$ & $F(2,180)=14.36, \mathrm{p}<0.01$ & $\mathrm{~T} 1>\mathrm{T} 2>\mathrm{T} 3$ \\
\hline
\end{tabular}

individual physiotherapists assisted with this study. All participating physiotherapists were visited or telephoned by the first author to ensure that they were fully aware of the recruitment conditions (patients) and that they were clear about when to distribute each questionnaire. They were asked to recruit men and women between 18 and 60 years of age with severe (minimum 21 days restriction of normal function/or sports involvement) acute musculoskeletal injuries, including soft tissue injuries and fractures. Any cases involving litigation or back pain were excluded. As a whole, the participating physiotherapists were organised to recruit sports participants and non-participants. Physiotherapists distributed sets of questionnaires three times over the course of rehabilitation as follows: at the initial physiotherapy appointment; at the midway point in treatment; and at the last physiotherapy appointment. Physiotherapists also completed a short questionnaire at each time point.

\section{MEASURES}

A questionnaire package solicited basic patient information, hours of sport and/or exercise involvement a week before injury, and asked patients to rate their injury severity on a five point Likert scale $(1=$ not severe; $5=$ very severe). In addition, the package included six visual analogue scales, corresponding to the six profile of mood states (POMS) ${ }^{23}$ subscales: anxiety, anger, depression, confusion, energy, and fatigue. Validation of the visual analogue scales confirmed that the six subscales displayed acceptable internal consistency, with Cronbach's $\alpha$ ranging from 0.83 to $0.90 .^{24}$ Using the Bland and Altman method, ${ }^{25}$ an acceptable level of agreement was found between the present visual analogue scales and the POMS $(\beta=0.09, F(1,188)=1.38, \mathrm{p}=$ $0.24)$. An additional visual analogue scale was included to provide a measure of a patient's perceived recovery. Physiotherapists rated injury severity on a five point scale, and estimated recovery on a visual analogue scale.

DATA ANALYSIS

Analysis focused on psychological responses to injury at three points in time, corresponding to the beginning (time 1), middle (time 2), and end of rehabilitation (time 3 ). The outcome variables examined were the six mood subscales and patient rated recovery. The principal aim of this study was to examine participants' emotional responses, as well as their perceptions of recovery, in terms of the number of hours of sports participation a week before injury. Given the number of actual and poten- tial analyses with this data set, $\mathrm{p}<0.01$ was adopted in all correlation, analysis of variance, and analysis of covariance statistics to provide greater protection against type I errors.

\section{Results}

Complete data at each sampling point for both patient and physiotherapist were available for 93 patients. The mean (SD) age was 35.61 (13.10) years; 55 were men and 38 women. On average, they were involved in sport and exercise for 5.63 (6.05) hours a week before injury. Their mean (SD) injury severity rating was 3.30 (1.11), and the analogous physiotherapists' rating was 3.39 (0.87); these ratings did not differ significantly.

OUTCOME VARIABLES: CHANGES OVER TIME

With the exception of self rated energy and recovery, which increased over time, all other outcome variables (anxiety, depression, confusion, anger, and fatigue) decreased as rehabilitation progressed. Analysis of variance showed that changes across time were significant. Post hoc analysis using the Newman-Keuls method disclosed that, in all cases, these temporal means differed significantly from one another (table 1).

ANALYSIS COMPARING NUMBER OF HOURS OF SPORTS INVOLVEMENT AND OUTCOME VARIABLES Correlations were undertaken between number of hours of sport and exercise involvement before injury and the following potential confounders: age, physiotherapist rated and patient rated injury severity, and length of formalised rehabilitation. A negative association was observed between age and the weekly time invested in sport and exercise $(r(92)=-0.28$, $\mathrm{p}<0.01)$; as would be expected, younger participants were more involved. No other significant correlations emerged. In addition, analysis of variance showed no significant differences between men and women with respect to the number of hours of sport and exercise involvement. Accordingly, subsequent analysis was by partial correlation controlling for age.

The only significant relations to emerge between sports and exercise involvement and the outcome variables, using the more stringent $\mathrm{p}<0.01$ criterion, were between number of hours of sport and exercise involvement and confusion at time $2(r(87)=0.27, \mathrm{p}<0.01)$ and time $3(r(87)=0.29, \mathrm{p}<0.01)$, and perceived recovery at time $3(r(87)=-0.27, \mathrm{p}<0.01)$. Athletes who were more involved were more confused in the middle and at the end of rehabilitation, and perceived their recovery to be less at the end of rehabilitation. 
ANALYSIS COMPARING THE HIGHEST AND LOWEST QUARTILES BASED ON NUMBER OF HOURS OF SPORTS PARTICIPATION A WEEK

The analysis reported so far may not have captured the particular adjustments to injury of highly committed sports people, as it treated sports and exercise involvement as a continuous variable. Accordingly, further analysis was conducted comparing the highest and lowest quartiles of involvement. The lowest quartile consisted of 23 people who exercised for one hour or less a week, whereas the 22 in the highest quartile dedicated eight hours or more a week to sport and exercise. One way analysis of variance was used to compare the lowest and highest quartile with respect to the previously identified potential confounders: age, physiotherapist rated and patient rated severity, and length of formalised rehabilitation. As may be expected, a significant group difference emerged for age $(F(1,43)=9.43, \mathrm{p}<0.01)$; those who invested eight hours or more a week were significantly younger (31.95 years) than those who invested one hour or less $(43.83$ years). A $\chi^{2}$ test comparing the number of men and women in each group was not significant.

Two factor analysis of covariance (groups, time), with age as a covariate, was used to compare the lowest and highest quartiles on the outcome measures. Largely in line with the outcome of the previously reported correlational analysis, the only significant groups main effect to emerge was for confusion $(F(1,41)=$ 9.39, $\mathrm{p}<0.01)$; the high participation quartile were more confused than the low participation quartile. All variables changed significantly over time, in accordance with the temporal pattern reported earlier, and no significant groups $\times$ time interactions were found.

\section{Discussion}

In line with previous research, injury had discernible emotional effects, ${ }^{36}$ and the negative emotional impact of injury diminished over the course of rehabilitation ${ }^{8-11}$ as self rated recovery improved. The average estimates of recovery by participants at time 1,2 , and 3 corresponded to about $40 \%, 60 \%$, and $80 \%$ of rehabilitation, and mean physiotherapist rated recovery scores were about $30 \%, 60 \%$, and $80 \%$ of rehabilitation. These data provide confirmation that assessments were, as planned, undertaken at approximately the beginning, middle, and end of rehabilitation. It is worth noting here that discharge occurs not at $100 \%$ recovery but at just under $80 \%$.

It was hypothesised that those who were more involved in sport before injury would exhibit a greater emotional response to injury and perceive their recovery to be less. Only confusion and recovery were associated with the number of hours of sports and exercise involvement; those more involved in sport before injury were more confused at the middle and end of rehabilitation, and perceived their recovery to be less by the end of formal rehabilitation.

The only published study that has explicitly explored the relation between number of hours of sports involvement a week before injury and emotional responses reported no significant relation between hours of sports involvement and any of the POMS emotions, ${ }^{11}$ although they did report a weak relation between hours of sports involvement and anger. Number of hours of sports involvement before injury was, in line with current findings, significantly related to age, with younger participants being more involved. However, age and anger were also correlated, thus it is possible that the putative relation between sports involvement and anger was a statistical artefact which arose from their common association with age. Accordingly, there is a precedent for the present failure to find widespread associations between the affective impact of injury and athletic status.

With regard to the association between sports involvement and confusion, those more involved in sport may have had greater information needs than those less involved, and experienced confusion when such needs were not met. Those more involved in sport and exercise may require more information about alternative activities to help maintain aerobic fitness. Similarly, they may require more rehabilitation specific information in order to regain their previous high level of physical functioning; highly committed athletes may be more confused at the end of rehabilitation because they know that they are still a long way from attaining the status they had before injury. This is supported in this study by the finding that those more involved in sport perceived their recovery to be less at the end of rehabilitation, compared with those less involved.

From a practical point of view, the present results suggest that, relative to injured nonathletes, injured athletes perceived a greater mismatch between current physical status and that before injury. It is possible that physiotherapists require training in order to appreciate more fully the information needs of the highly involved athlete. Researchers have increasingly acknowledged that the physiotherapist is ideally placed to provide psychological support to injured athletes. ${ }^{26}$ However, several studies have found that sports injury rehabilitation personnel would prefer a greater emphasis on psychological factors in their professional training. ${ }^{27-29}$

Recent work in Australia has resulted in a proposed psychoeducational curriculum for the training of sports injury rehabilitation personnel. ${ }^{30}$ The professional training of sports injury rehabilitation personnel in the United Kingdom does not routinely include the level of training proposed in the Australian psychoeducational curriculum; although cultural differences must be acknowledged, the Australian prototype may offer a useful framework for future work in the United Kingdom.

This study is arguably more wide ranging than previous studies of injured athletes ${ }^{3-11}$ It is the first substantial study to include, as a control, people who were relatively uninvolved in sport and exercise. However, as with any study, it is not without shortcomings. Firstly, a number of authors have suggested that the POMS may not adequately reflect our mood 
state and that it may be more appropriate in future quantitative work to use the PANAS scale. ${ }^{31}$

Secondly, as with any quantitative research, this study may be criticised for producing a somewhat fragmented and imprisoned representation of the injury experience. Further qualitative work is needed to include the subjective experiences of the injured athlete. This would permit a more detailed insight into the way patients appraise the injury episode and the emotions experienced. Both qualitative and quantitative research exploring potentially more subtle differences between the psychological reactions to chronic versus acute injuries may prove particularly illuminating.

In conclusion, the consistent findings relating to confusion and perceived recovery reinforce the importance of assessing reactions to re-entry. Recent qualitative findings that re-entry was often associated with fear of reinjury and loss of sports confidence ${ }^{12}$ further underlines the need to extend assessment to the re-entry phase in future research.

1 Nicholl JP, Coleman P, Williams BT. Injuries in sport and exercise: main report. London: Sports Council: 1993.

2 Nicholl JP, Coleman P, Williams BT. The epidemiology of sports and exercise related injury in the United Kingdom. Br f Sports Med 1995;29:232-8.

3 Chan CS, Grossman HY. Psychological effects of running loss on consistent runners. Percept Mot Skills 1988;66:875loss

4 Leddy $\mathrm{MH}$, Lambert MJ, Ogles BM. Psychological consequences of athletic injury among high-level competitors. Res Q Exerc Sport 1994;65:347-54.

5 McGowan RW, Pierce EF, Williams M, et al. Athletic injury and self diminution. I Sports Med Phys Fitness 1994;34: 299-304.

6 Pearson L, Jones G. Emotional effects of sports injuries: implications for physiotherapists. Physiotherapy 1992;78: 762-70.

7 Smith AM, Stuart MJ, Wiese-Bjornstal DM, et al. Competitive athletes: preinjury and postinjury mood state and selfesteem. Mayo Clin Proc 1993;68:939-47.

8 Crossman J, Gluck L, Jamieson, J. The emotional responses of injured athletes. New Zealand Fournal of Sports Medicine 1995;23:1-2.

9 McDonald SA, Hardy CJ. Affective response patterns of the injured athlete: an exploratory analysis. Sport Psychologist

10 Quackenbush N, Crossman J. Injured athletes: a study of emotional responses. Fournal of Sport Behaviour 1994;17: 178-17.
11 Smith AM, Scott SG, O'Fallon WM et al. Emotional responses of athletes to injury. Mayo Clin Proc 1990;65:38-

12 Johnston LH, Carroll D. The context of emotional responses to athletic injury: a qualitative analysis. Fournal of Sport Rehabilitation 1998;7:206-20.

13 Rose J, Jevne RFJ. Psychosocial processes associated with athletic injuries. Sport Psychologist 1993;7:309-28.

14 Udry E, Gould D, Bridges D, et al. Down but not out: athlete responses to season-ending injuries. Fournal of Sport and Exercise Psychology 1997;19:229-48.

15 Brewer BW. Review and critique of models of psychological adjustment to athletic injury. Fournal of Applied Sport Psychology 1994;6:87-100.

16 Smith AM. Psychological impact of injuries in athletes. Sports Med 1996;22:391-405.

17 Scanlan TK, Carpenter PJ, Schmidt GW, et al. An introduction to the sport commitment model. Fournal of Sport and Exercise Psychology 1993;15:1-15.

18 DiLorenzo TM, Bargman EP, Stucky-Ropp R, et al. Long-term effects of aerobic exercise on psychological outcomes. Prev Med 1999;28:75-85.

19 Weyerer S, Kupfer B. Physical exercise and psychological health. Sports Med 1994;17:108-16.

20 Brewer BW. Self-identity and specific vulnerability to depressed mood. F Pers 1993;61:343-64.

21 Kleiber DA, Brock SC. The effect of career-ending injuries on the subsequent well-being of elite college athletes. Sociology of Sport Fournal 1999;9:70-7.

22 Little JC. The athlete's neurosis: a deprivation crisis. Acta Psychiatr Scand 1969;45:187-97.

23 McNair DM, Lorr M, Droppleman LF. Manual for the profile of moods states. San Diego: Educational and Industrial Testing Service, 1971.

24 Johnston LH. The temporal and situational context of athletes' emotional responses following injury. $\mathrm{PhD}$ thesis, University of Birmingham, 1997.

25 Bland JM, Altman DG. Statistical methods for assessing agreement between two methods of clinical measurement. Lancet 1986;8:307-10.

26 Wiese-Bjornstal DM, Smith AM. Counseling strategies for enhanced recovery of injured athletes within a team approach. In: Pargman D, ed. Psychological bases of sport injuries. Morgantown, WV: Fitness Information Technology, 1993:149-83.

27 Ford IW, Gordon S. Perspectives on the psychological curricula in professional training programs of sport physiotherapists and sport/athletic trainers: a cross-cultural survey. Proceedings of the annual meeting of the Association for the Advancement of Applied Sport Psychology, Lake Tahoe, NV, 1994.

28 Gordon S, Milios D, Grove JR. Psychological aspects of the recovery process from sport injury: the perspective of sport physiotherapists. Aust $\mathcal{F}$ Sci Med Sport 1991;23:53-60.

29 Wiese DM, Weiss MR, Yukelson DP. Sport psychology in the training room: a survey of athletic trainers. Sport Psychologist 1991;5:15-24.

30 Gordon S, Potter M, Ford IW. Toward a psychoeducational curriculum for training sport-injury rehabilitation personnel. Fournal of Applied Sport Psychology 1998;10:140-16.

31 Watson D, Tellegen A. Toward a consensual structure of mood. Psychol Bull 1985;98:219-23.

\section{Take home message}

Injury appears to have largely the same affective impact for those with minimal and considerable involvement in sport and exercise before injury. Those who are move involved register higher levels of confusion and perceive their recovery to be less at the end of rehabilitation. 